\title{
Solving Traveling Salesman Problem Using Cuckoo Search and Ant Colony Algorithms
}

\author{
Luma Salal Hasan
}

\section{Dept. Multi-Media / College Computer Sc. \& Information Technology University of Al-Qadisyiah \\ Luma.Hasan@qu.edu.iq \\ Dr.luma.hasan@gmail.com}

Recived : $14 \backslash 1 \backslash 2018$

Available online : $3 / 4 / 2018$

DOI: $10.29304 / j q c m .2018 .10 .2 .377$

\author{
Revised : $4 \backslash 2 \backslash 2018$
}

Accepted : 261212018

\begin{abstract}
Optimization is a method that is used from economic to design. The best tools available are very important to be utilities .when there is some randomize nature value that's depend in the algorithm is called stochastic. Algorithm with stochastic partitions are often named heuristic or meta heuristic recently.

Traveling salesman problem (TSP)is hard a combinatorial optimization problem that leads to find the best tour for the person . this problem can be applicator in many different area such as DNA fragments, planning and logistics. There are many algorithm that is used to solve this problem.

In this paper, Ant colony optimization (ACO) is the first algorithm that is applied which depending on the ant colonies law for finding the best tour of TSP .

The other algorithm that is performed, is cuckoo search (CS) that satisfy the law of brood parasitism of some cuckoo specie to find the best tour of the same problem.

Compare between two algorithms of meta heuristic for six cities with different parameter's value to evaluate the result . conclude that the CS performance is better than ACO with speed convergence.
\end{abstract}

Keywords: Ant colony optimization, Cuckoo search algorithms, Traveling salesman problem. 


\section{Introduction}

optimization is the achieving the best possible result under circumstances. It design, built then maintenance, engineers have to take decisions. The goal of decisions is either for minimize the efforts or maximize the benefit of it which can be modeled as a function of certain design variables. Then ,the optimization can be defined as the process of finding the conditions that lead to maximize or minimize the value of a function[1].

Exploration and explotation by using search locally and randomize values are the important components of all meta heuristic algorithms. It firstly started with one or more solutions then tested steply on a sequence of solutions to reach the optimal solution .

The most popular meta heuristic algorithm are Genetic Algorithm(GA), Tabu Search(TS), Simulated Annealing(SA), Ant Colony Optimization(ACO), Particle Swarm Optimization(PSO), Bee Colonies Optimization (BCO), Monkey Search Algorithm(MS), Harmony Search Algorithm(HS), Fire Fly Algorithm(FA), Intelligent Water Drops(IWD) and Cuckoo Search (CS) [2].

Most of there, are mimicking successful feature in biological ,physical or sociological system. The success of these methods for solving combinatorial optimization depending on many features[3 ].

Travel salesman problem ,firstly formulated as a mathematical problem in 1930's and most studied in theoretical computer science mathematical and engineering applications. The important parameters of this problem I cost ,time, path are optimized. Recently, applicator in computer network ,mail carries delivery trucks and airways[4].

ACO are used by Macro Dorigo in 1992.it modified to used probabilistic to solve the TSP by graph in 1997[5], which are described in section 2.

Cuckoo search was inspired by the "obligate broad parasitism" of many host cuckoo's birds of the other species. It proposed by Yank[3],that described in section 3 briefly.

\section{2 .Ant Colony Algorithm}

Are depend on the behavior of natural ant colonies. Real ants find shortest path leading from the nest to food sources by laying a chemical compound which known as pheromone on the ground. A number of artificial ants construct a solutions randomly. Each ant selected probabilistic to follow a path with rich pheromone[6].

When used on TSP this means artificial ant moves from city to another on a graph( count the edges travelled accumulating then the pheromone trial left behind by another[7].

The algorithm steps are:-

1.Generate coordinate of cities randomly depending on the number of cities.

2.Each ant choose city i randomly then move to city $\mathrm{j}$ using probabilistic transition $\operatorname{rule}\left(\mathrm{S}_{\mathrm{ij}}{ }^{\mathrm{k}}(\mathrm{t})\right)$, to built the tour using the following equation[8].

$\left.\mathrm{S}_{\mathrm{ij}}{ }^{\mathrm{k}}(\mathrm{t})=\left(\mathrm{w}_{\mathrm{ij}}(\mathrm{t})\right)^{\alpha}\left(1 / \mathrm{d}_{\mathrm{ij}}\right)^{\beta}\right) /\left(\sum\left(\mathrm{w}_{\mathrm{il}}(\mathrm{t})\right)^{\alpha}\left(1 / \mathrm{d}_{\mathrm{il}}\right)^{\beta}\right)$ if $\mathrm{j} \in \mathrm{J}_{\mathrm{k}}(\mathrm{i}) \ldots \ldots .1$

If $\mathrm{j} \notin \mathrm{J}_{\mathrm{k}}(\mathrm{i})$ then $\mathrm{S}_{\mathrm{ij}}{ }^{\mathrm{k}}(\mathrm{t})=0$

Where

$\mathrm{d}_{\mathrm{ij}}$ is the distance between city $\mathrm{i} \& \mathrm{j}$, is computed by the equation

$\mathrm{d}_{\mathrm{ij}}=\sqrt{\left(z_{i}-z_{j}\right)^{2}+\left(y_{i}-y_{j}\right)^{2}}$

$\mathrm{J}_{\mathrm{k}}(\mathrm{i})$ is a set of cities that remain to be visited when the ant is at city $i$.

$\alpha, \beta$ are positive parameters that control the weight of pheromone trial which computed for each tour by using this equation.

$\mathrm{w}_{\mathrm{ij}}(\mathrm{t}+1)=(1-S) w_{i j}(t)+\Delta r_{i j}(t)$
$\Delta w_{i j}(t)=\sum_{k=1}^{n} \Delta r_{i j}^{k}(t)$
$\Delta r_{i j}^{k}(t)=\left\{\begin{array}{rr}A / D_{k} & \text { if }(i, j) \in \text { tour by ant } k \\ 0 & \end{array}\right.$

Where (1-s) is a pheromone decay parameter $0<\mathrm{S}<1$ it's used when the ant choose a city to move it (evaporation trial), $\mathrm{n}$ is the number of ants, $\mathrm{D}_{\mathrm{k}}$ is the length of the tour by ant $\mathrm{k}$ and

A is an arbitrary constant.

3. Add cities one by one until get the complete visited cities.

4. Find the best tour for each iteration. 


\section{Cuckoo Search Algorithm}

Is a modern new meta-heuristic search algorithms that proposed by Xin-She Yang \& Snash Deb in 2009[3] ,"inspired by the broad parasitism of a lot of cuckoo bird species". It depending on three laws .firstly, each cuckoo put egg only one at each time in the host nest randomly, while secondly, the best nest which have the best quality of eggs that wining out to the nest generation .lastly, the number of host is fixed the probability of egg discovery by the host bird is $\mathrm{P}_{\mathrm{a}}$ $\in[0,1][4]$.
The bird alien the eggs on the nest that it's belong to their. Then the bird build the new nest on another place. The flowchart below in figure.1are described the steps for solving TSP.

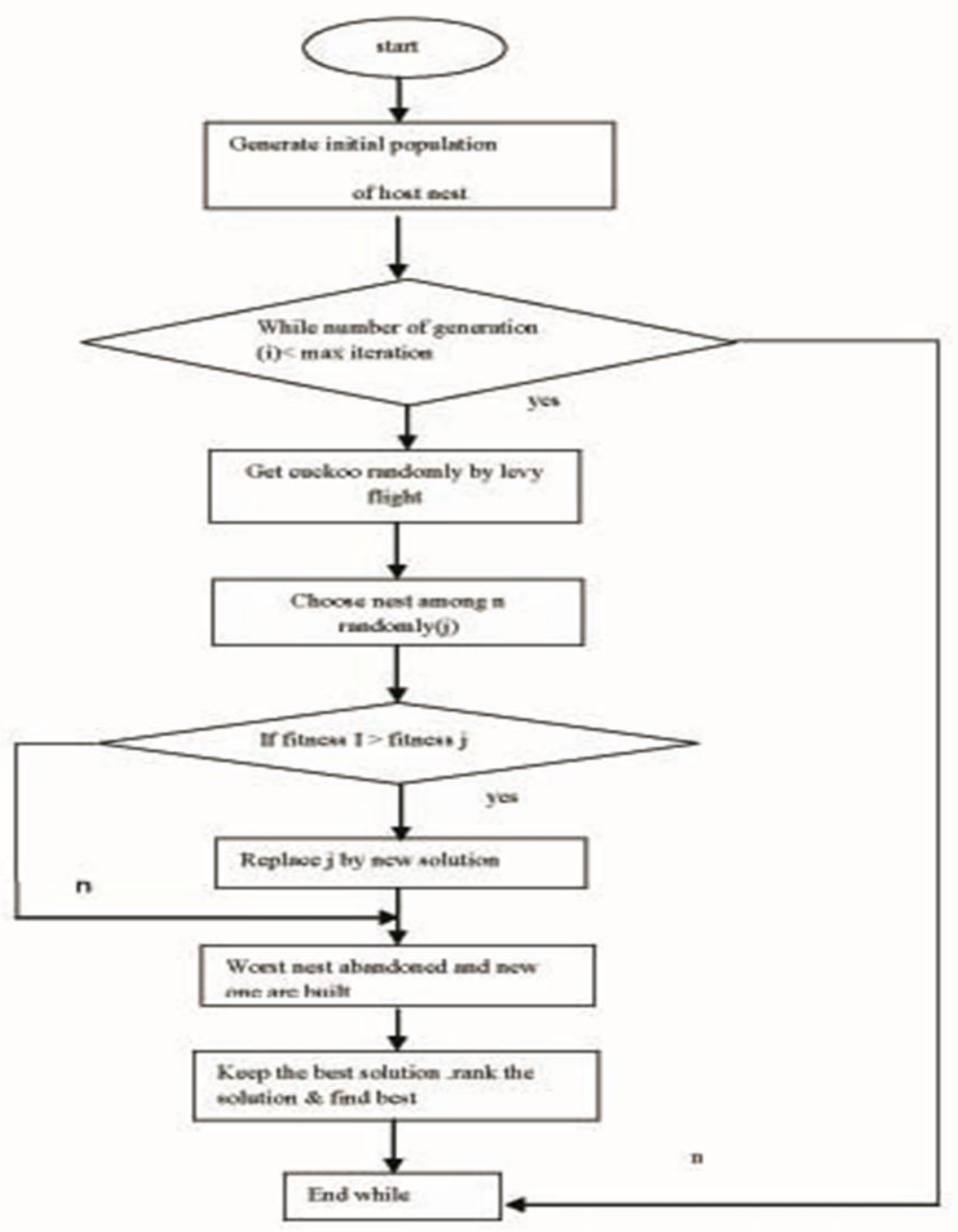

Figure 1.Flowchart for solving TSP by CS 


\section{Experimental Results}

In order to evaluate the behavior of cuckoo search and ant colony algorithms by using matlab ver.7, using several values of parameters for six of cities for TSP.

Firstly, apply the ACO with the value of $\alpha=1, \beta=4$ with six cities. In the figure 2 , show the tour city's number for six generation.

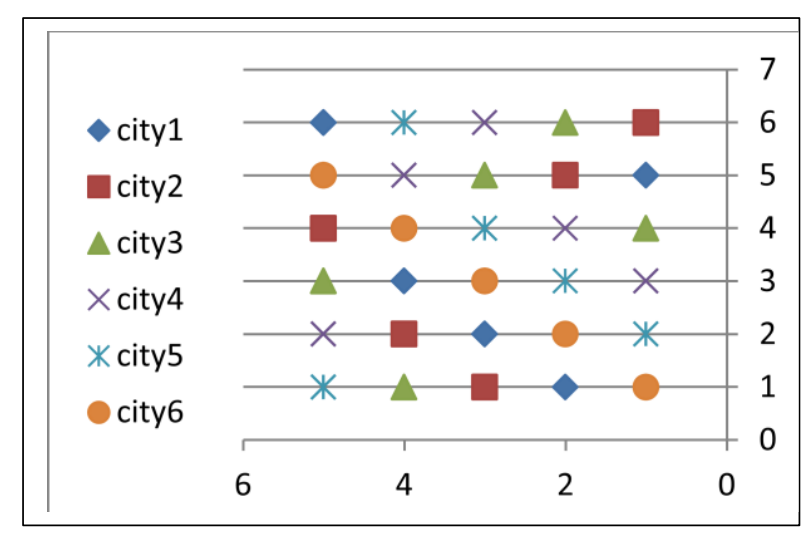

Figure 2.tour for six cities

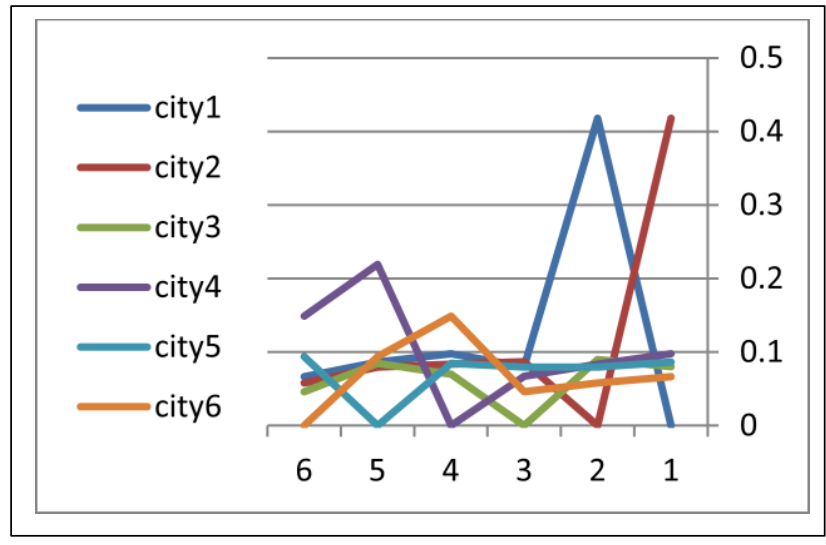

Figure 3. best fitness for the cities

While in figure 3, display the best fitness for the same tour that show in figure.2. With the best fitness is 0.0930 for the tour with city's number (5 64321 5).

In figure below show the tour for the same number of cities with $\alpha=1$ and $\beta=2$.the best tour are(2 563 142 ) with best fitness $=0.0940$

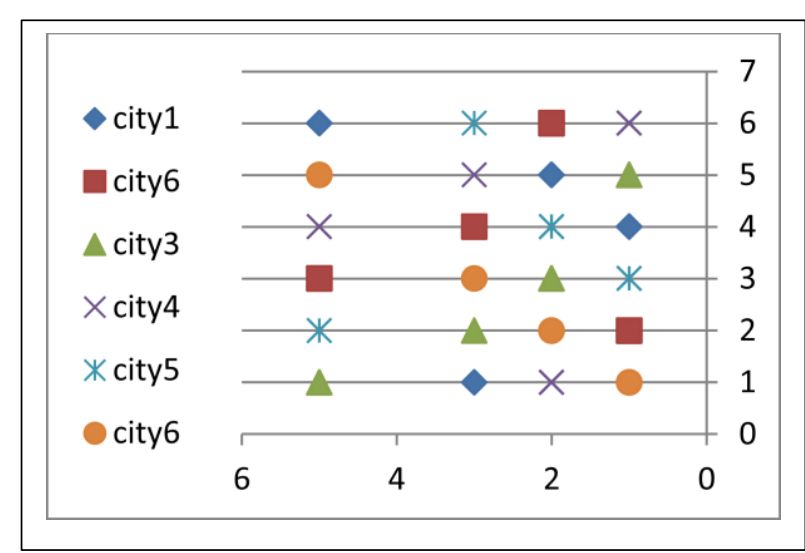

Figure 4. tour for six cities with $\alpha=1$ and $\beta=2$

From the above figures, deduce that, the tour consume more time because the tour most start and ended with same cities. Also, when decrease the value of $\beta$, the value of best fitness are increase. When perform CS for the TSP with six cities, the tour are display in figure 5 .

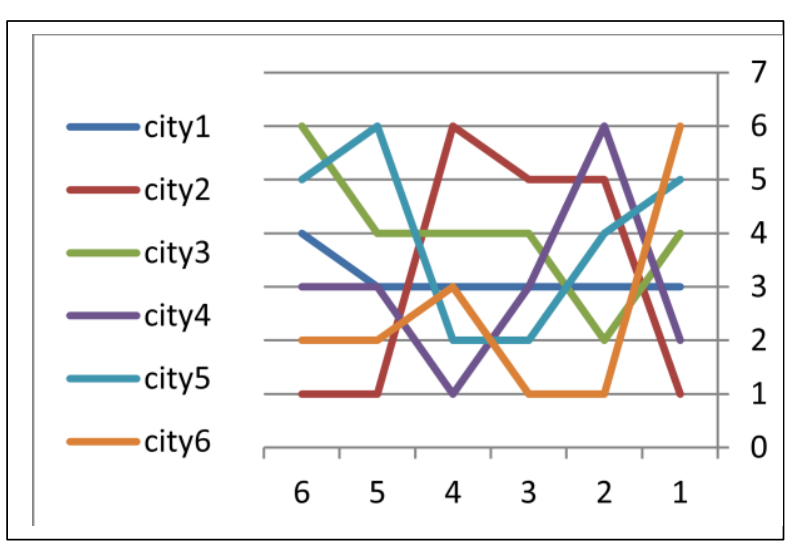

Figure 5, CS tour for six cities 


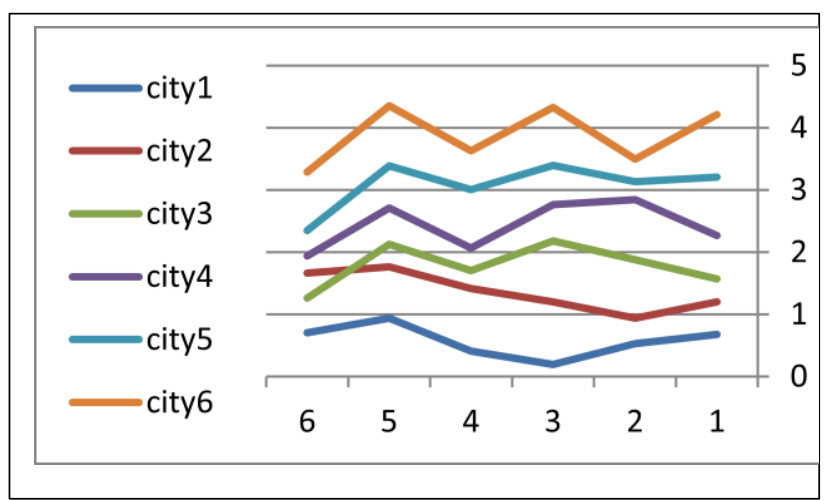

Figure 6, the fitness for the tour's cities

The best fitness for the tour are notice in figure 6.the best tour are 136254 .

From the figures above, deduce that the best solution quality is better when used CS algorithm for TSP with consuming time less than when compare with ACO. This effect on the convergence is quickly occurs in this algorithm.

\section{Conclusion}

This paper presents two approach of meta heuristic search algorithms for solving traveling sales man problem that based on ant colony and cuckoo search algorithms .from our experimental results, it has been shown to compare between two meta heuristic algorithms. ACO is simple in computation and the tour construction consume more time depending on the condition each tour must start and ended with the same cities. Also, when decrease the value of $\beta$, the value of best fitness are increase. but the CS is better performance to find the best tour quickly compared with other by consuming time less than when compare with ACO. This effect on the convergence speed for searching the solution is rapidly compared with it.

The cuckoo search algorithm is more efficient than ant colony in terms of ability for finding the better solution .

\section{References}

[1] A.Astolfi, Optimization an introduction, Chapter 2, Sept.2006.

[2] H. Eldem \& E.Vlker, Application of ACO for the solution of 3 dimensional cuboid structures ,journal of computer and communication, Vol.2, PP. 99-107, 2014,

[3] Xin-She Yang \& Snash Deb, Cuckoo search via levy flights, world congress on nature \& biologically inspired computing NaBIC2009, IEEE, pp.210-214, 2009

[4] A.S.Tawfik \& A.A.Badr, I.F.Rahman, One rank cuckoo search algorithm with application to algorithmic trading system optimization, International journal of computer applications,Vol.64,No.6,pp.30-37, Feb.2013.

[5] Dorigo,M.\& Gambarella L.M , Ant colony system, A cooperative learning approach to the TSP, IEEE on evolutionary computation, PP. 53$66,1997$.

[6] M.Gendreau \& J.Y.Potvin, Metaheuristics in combinatorial optimization, Canada,H3C 3 J7.

[7] B.Lil, L.Wang, and W.Song, Ant colony optimization for the traveling salesman problem based on ants with memoey, $4^{\text {th }}$ International conference on natural computation IEEE,pp.496500,2008 .

[8] Zar, Hlaing \& M.A.Khine, TSP problem by using improved ant colony optimization algorithm, Inter. Journel of information and education technology ,Vol.1,No.5, Dec.2011. 


\title{
حل مشكلة البائع الجوال باستخدام
}

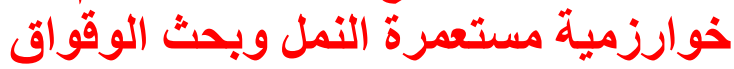

\author{
لمى صلال حسن \\ جامعة القادية / كلية علوم الحاسوب وتكنولوجيا المعلومات / قسم الوسائط المتعددة \\ Luma.Hasan@qu.edu.iq \\ Dr.luma.hasan@gmail.com
}

(المستخلص : (1) (2)

التحسين هو طريقة مستخدمة في الاقتصاد الى التصميم.و تعتبر افضل اداة مستخدمة عندما تتوفر

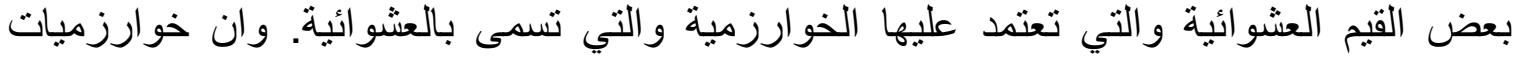

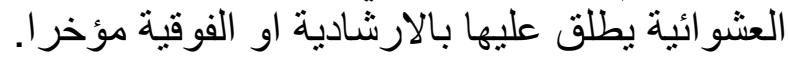

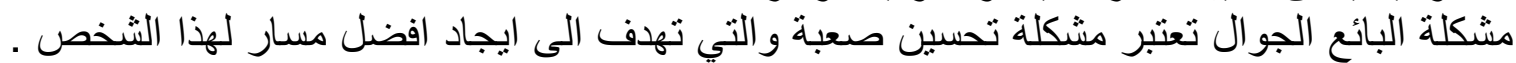

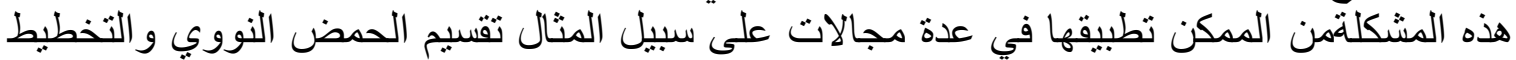
و الخدمات اللوجستية. في هذا البحث ، تم تطبيق خوارزمية تحسين مستعمرة النمل و التي تعتبر خوارزمية عشو ائية والتي

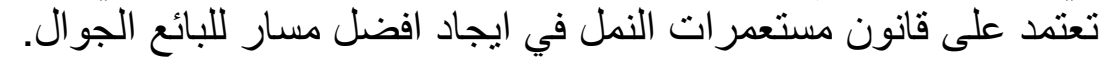
ثم تم تطبيق خوارزمية بحث الوقواق والذي يعتمد على قانون حضانة بيض الفقس لبعض عش

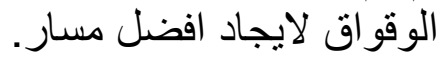
تمت مقارنة الخوارزميتين لستة مدن لتقييم النتائج تم التوصل التى الى ان اداء خوارزمية بحث الوقواق

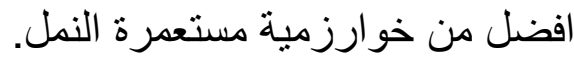

